\title{
Nanocytology vs. Immunohistochemistry of Intestinal Colonocytes to Assess the Risk of Colon Cancer based on Field Cancerization - A Preliminary Report
}

\author{
Myron Arlen ${ }^{\bowtie}$, Olga Saric, XuePing Wang, Alex Dubeykovskiy, Philip Arlen \\ Division Surgical Oncology, North Shore University Hospital, Manhasset, NY, and Precision Biologics, Great Neck, NY, USA.
}

$\bowtie$ Corresponding author: marlen@neogenix.com.

(ㅇ Ivyspring International Publisher. This is an open-access article distributed under the terms of the Creative Commons License (http://creativecommons.org/ licenses/by-nc-nd/3.0/). Reproduction is permitted for personal, noncommercial use, provided that the article is in whole, unmodified, and properly cited.

Received: 2012.10.31; Accepted: 2013.0I.II; Published: 2013.02 .02

\begin{abstract}
The ability to define malignancy in its earliest stages of development is an essential part of any program aimed at attempting to cure the malignant condition. In terms of colon cancer various approaches have been employed to define the transformation of colonocytes as they progress to the fully malignant phenotype. Approaches ranging from nanocytology to mass spectroscopy have been utilized with limited success.

Our group at Precision Biologics has been able to define three distinct immunogenic proteins, most oncofetal in origin, which are expressed to various degrees in colon cancer and are essentially absent from normal colon tissue. Monoclonal antibodies (mAbs) have been developed against these tumor associated antigens (TAA), which is NPC-I, 3I.I and I6C3. Each, have shown significant ADCC in the presence of the tumor cells grown in culture. Studies were performed to clarify at what stage in the development of the colon cancer do such TAA proteins begin to be expressed. Utilizing Immunohistochemistry (IHC) with the mAbs targeting the TAA's, we have been able to demonstrate that such antigens appear in the cytoplasm as early as 6 or more months prior to the phenotypic appearance of malignancy utilizing H\&E staining.

Kits containing these colon $\mathrm{Ca}$ monoclonals from our lab, as well as positive and negative controls have been produced for use in the operating room to examine colonocytes at the margin of resection following colectomy; this in order to assure that transforming cells are not incorporated into an anastomosis. We have also been able to demonstrate that premalignant cells as well as those cells present in a fully malignant lesion do shed their antigens into the lumen of the bowel. As such, we have been able to show that a simple "office" stool ELISA can predict with a high degree of accuracy whether a premalignant polypoid lesion, a fully malignant adenocarcinoma or a totally normal colon free of any neoplastic process is present and thus decide on the need for or not, of performing colonoscopy.
\end{abstract}

Key words: Colon monoclonal antibodies, Immunohistochemistry, transforming colonocytes, Stool ELISA.

One of the major issues of concern to the surgeon as well as pathologist at the time of colon resection, is in defining adequate margins of resection. This problem exists for the most part, when left sided colon malignancies are resected at or near the peritoneal reflection. Here, most surgeons accept distal margins free of disease at from 2 to $5 \mathrm{~cm}$. below the lower margin of the neoplasm to be resected. Distal margins 
are then carefully examined by the pathologist, usually in the OR, to perform the needed frozen sections for careful inspection of the specimen. When it is reported that colonocytes present at the margins of resection appear normal, an anastomosis is then carried out. In spite of this approach to assure that the tumor being resected has been totally removed, a local suture line recurrence of from $10-15 \%$ can be anticipated within two years of the surgery that had been performed. In the present report we describe an immunohistochemical procedure, readily available for use on frozen section material, to define which cells interpreted as normal, have actually undergone genotypic transformation toward clinical malignancy.

Even though tumor cells may have spread into the circulation prior to tumor resection, resulting in synchronous or metachronous metastasis arising fromm the primary colon lesion, optimization of margins of resection at the time of the surgical procedure is essential to minimize or totally prevent anastomotic recurrence. A number of explanations have been given for this form of local recurrence, including re:emergence of malignant stem cells at the suture line or even retrograde spread of malignant cells that remained in the mesenteric lymphatic channels following the surgical procedure.

We believe that such concepts related to recurrence, fail to recognize premalignant oncogenic transformation that has already taken place in normal appearing cells such as colonocytes. Such cells have the potential to compromise a surgical procedure, resulting in local recurrence. The development of monoclonal antibodies that were shown to be capable of recognizing cancer proteins being expressed in both fully malignant as well as premalignant cells, originated in a study employing therapeutic immunogenic proteins isolated from colon cancer. These proteins were employed as a vaccine to control Dukes C2/D lesions undergoing resection. Here, marked improvement in $5 \mathrm{yr}$. survival was noted in the vaccine treated group over surgery alone. This beneficial vaccine response was studied to define mechanisms. When a final analysis of the data was reviewed, it proved that this improved survival was mainly due to the production of high titers of a specific IgGlantibody that defined proteins expressed in the tumor and then targeted these immunogenic proteins that appeared within the colon malignancy. In this study employing a colon cancer vaccine to enhance the immune response, little efficacy was found to be associated with the onset of cell mediated immunity. Based on these findings we approached the issue of defining the immunogen as a marker and such markers then having the capability of being targeted for apoptosis. We now utilized those tumor antigens derived from colon cancer to develop a repertoire of mAbs (monoclonal antibodies). Three mAbs were produced, sequenced and then cloned as murine and chimeric antibodies that targeted three distinct tumor proteins essentially expressed in all colon cancers examined (Fig. 1).

In approaching FDA regarding use of these monoclonals for possible therapy against metastatic colon tumors, it was necessary to evaluate a large array of normal colon and other tissues to define whether any of the corresponding antigens developed against colon cancer were expressed in normal tissue. On completion of the study, no normal tissues could be shown to express such immunogenic tumor protein antigens.

A major issue followed as to when in the clinical development of a malignant lesion such as colon cancer, did tumor protein become expressed and that such antigen be readily identified. This analysis evaluated antigen expression ranging from what was interpreted as the initial genotypic transformation of the colon cells to the phenotypic appearance of malignancy. Our evaluation of numerous histologic sections employing immunohistochemistry with a combination of the three monoclonals targeting immunogenic tumor proteins looked at normal tissue in patients without disease, those where the earliest polypoid changes were taking place, and samples from normal colonocytes adjacent to a large array of early and late malignancies. The information obtained, suggested that the presence of, or expression of these immunogenic tumor proteins that would represent transformation to the malignant phenotype, could be detected at the earliest onset of the genotypic state of malignancy when the colonocytes still appeared normal.. This event suggested that tumor protein could be identified somewhere between 4 and 6 months before phenotypic features were noted by the pathologist using H\&E for the staining procedure, We also noted that in many cases of colon cancer, a field effect extending from the primary tumor to several $\mathrm{cm}$. past the tumor was present. Here, normal appearing colonocytes expressing proteins similar to ones defined in the primary malignant lesion were present. (Fig. 2)

Of significance was the need to ascertain whether the presence of such cells noted at the margins of resection represented inadequacy of tumor removal, and as a result the development of anastomotic recurrence. We did have the opportunity to evaluate several instances where anastomotic recurrence was seen and where the original margins called normal did reveal tumor protein expression. In such 
cases where recurrence arose, we now interpreted such findings as being based on the inclusion of transforming cells in the suture line of the reconstructed bowel. A major retrospective study as such is underway to confirm statistically that such transforming cells do serve as the causative factor for disease reappearing in the suture line.

Alternative approaches have been devised to define transforming colonocytes. Damania et.al (1), described partial wave spectroscopic (PWS) microscopy as a means of assessing the presence of abnormal colonocytes as a cause of recurrence developing in the bowel suture line post surgery. This microscopic procedure was developed to resolve the same issues that we have had in terms of the reason for recurrent anastomotic lesions and how to prevent them. It was noted that "this approach provides a potential prescreening strategy for one of the major issues of concern to the surgeon and pathologist, in defining adequate margins of resection at the time of performing a colon resection". This problem exists for the most part, when left sided colon malignancies are resected at or near the peritoneal reflection. Most surgeons as noted, accept distal margins free of disease from 2 to 5 $\mathrm{cm}$. below the lower margin of the neoplasm to be resected. Distal margins are carefully examined on frozen section by the pathologist, usually standing by in the OR. When it is reported that colonocytes present at the margins appear normal by standard histologic procedures, an anastomosis is carried out.

We have utilized in contrast to what was described as the "PWS microscopy" approach, a more readily available technique to evaluate oncogenic transformation. We employ direct targeting of tumor protein by an relatively simple immunoperoxidase procedure with specific anti TAA IgG's This staining procedure appears to define malignant transformation that might be occurring in normal appearing colonocytes. Here the monoclonal antibody binds to the immunogenic proteins being expressed in these transforming cells. Monoclonal antibodies (NPC-1, 31.1 and 16C3) produced against the corresponding colonic tumor proteins were shown to be highly effective both in diagnosing the presence of markers for characterization of the malignancy as well as guiding an immune therapeutic approach to target colon cancer cells via ADCC (antibody dependent cell cytotoxicity) $(3,4)$.

The specific Immunogenic proteins associated with colon cancer were first described by Hollinshead (2) and later characterized by our group at Precision Biologics. The 3 antigens identified in the malignant bowel lesion appear related to oncofetal proteins necessary to help the fetus in the maturation of the bowel. The NPC- 1 monoclonal as well as others targeting the immunogenic tumor proteins were derived from pooled allogeneic antigen derived from colon cancer. After partial purification, the antigen preparation was used to induce arrays of hibridomas which after screening, resulted in the 3 specific monoclonals that appeared to target antigen in colon carcinoma. The NPC-1 antibody appeared to be associated with a modified (post translational) or possibly mutated form of MUC5ac, 31.1, with a modified form of A33 of Olds and 16C 3 was associated with CEAcam 5 and, 6. Those antibodies commercially available for defining the 3 nonmutated oncofetal antigens did not cross react with the mutated derivatives that have immunogenic properties and that we have been able to characterize. These commercial monoclonals as such have neither therepautic nor diagnostic capabilities. The monoclonals we employ, appear to be related to altered core peptides of the immunogenic TAA proteins

Our group had the opportunity to examine 60 normal colon biopsies taken adjacent to an array of colonic neoplasms and detected tumor antigen being expressed in 9 of the cases. This is roughly the incidence of anastomotic recurrence seen following low anterior resection where there might have been failure to identify the early transformation of colonocytes on a genotypic basis. A rapid IHC double monoclonal antibody staining study has been developed that we plan to be used in performing a more detailed study of margins of resection taken within a limited distance $(2-5 \mathrm{~cm})$ for the colon cancer primary. We are not sure whether a field effect arises in the bowel secondary to viral or carcinogenic transformation and a malignant process develops in the field effect or whether secondary to the appearance of a malignant lesion, a corresponding field effect around the tumor ensues. Obviously during the process of defining field effect, if antigen expression is noted in resected margins, further resection will be suggested. Two studies are in progress. A retrospective one, to look at a number of recurrences that appeared in the suture line 1-2 years following surgery. A second study will prospectively evaluate patients undergoing surgery where margins will then be evaluated by frozen section to help the pathologist clearly define normalcy in the margins remaining as part of the retained bowel.

As the concept of antigen expression in malignancies came to fruition, It seemed important to define whether premalignant cells as with their mature counterparts that are expressing antigen, shed their tumor proteins into the bowel lumen.

In a manner similar to what is noted when the fully malignant tumors shed tumor antigen into the 
serum (Fig.3) we undertook examining stools samples, a site for the accumulation of possible shed antigen. A small study was performed, the results being noted in Fig.4. For the most part, the stool ELISA as performed in this study, did detect shed antigen from both tumor as well as premalignant lesions. Such a

\section{H\&E on colon ca case \#2}

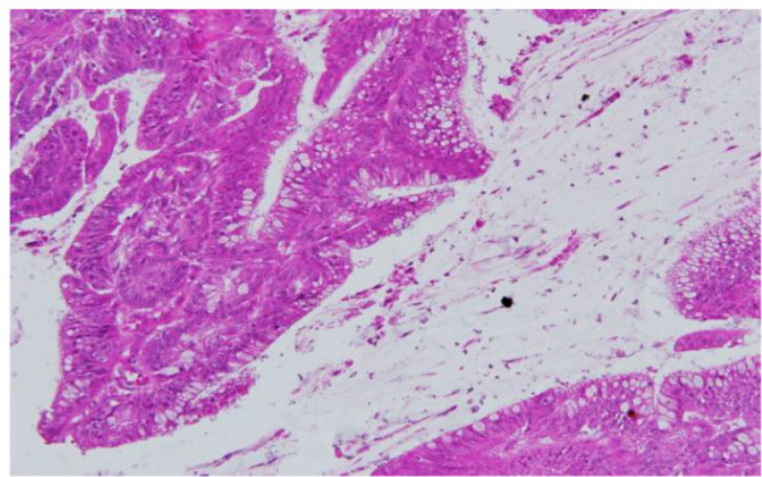

procedure appears sensitive and can suggest with a fair degree of accuracy whether the bowel is or is not free of pathology.

It is anticipated that this procedure will eventually replace the stool guaiac test for possible defining the presence of malignant pathology within the colon.

\section{1 at $5 \mathrm{ug} / \mathrm{ml}$ on colon ca case \#2}

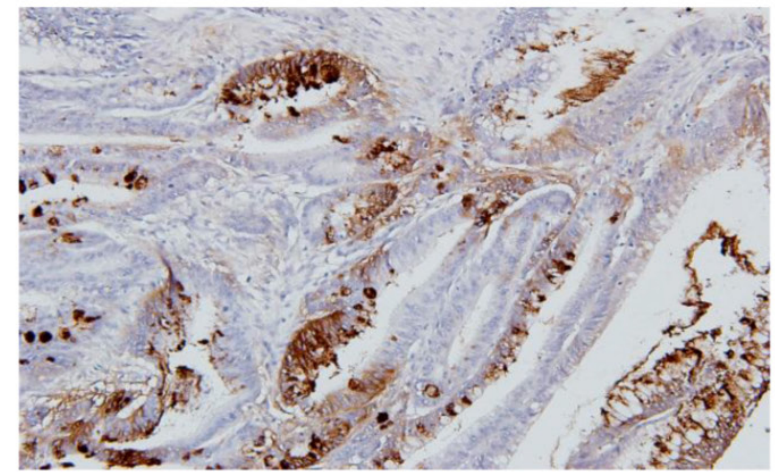

Fig I. Colon cancer stained with H\&E and then followed by IHC with mAb 3I.I.

\section{H\&E of normal mucosa adjacent to colon ca. case \#1}

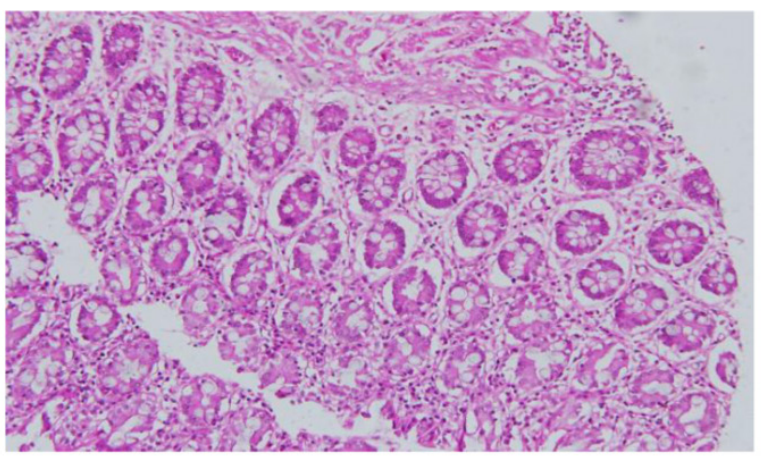

\section{NPC1-C $5 u \mathrm{~g} / \mathrm{ml}$ used for IHC in case \#1 which was read as benign}

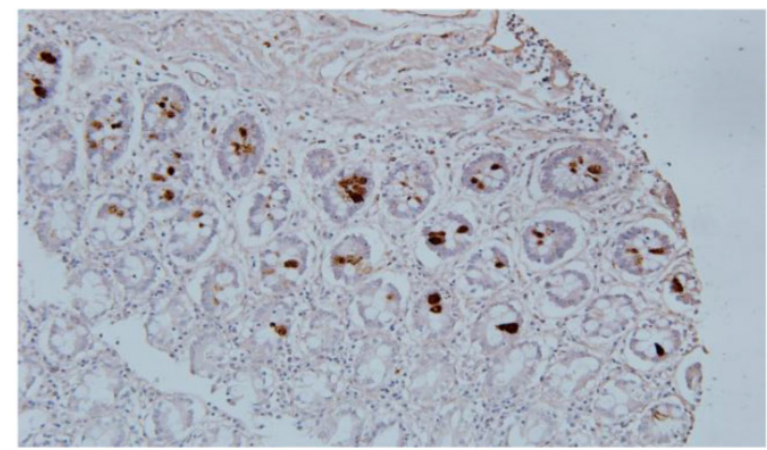

Fig 2. Normal colonocytes seen expressing tumor antigen defined by monoclonal antibody NPC-I. 


\section{Double stain 31.1 Red NPC1-C Dark brown}

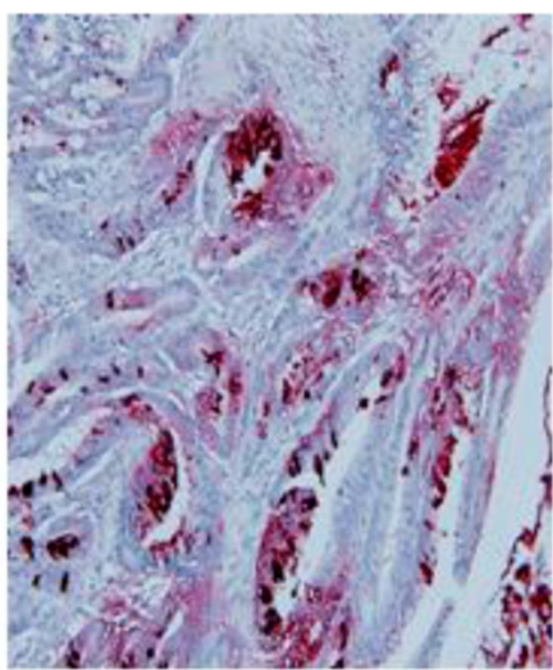

$20 \mathrm{X}$

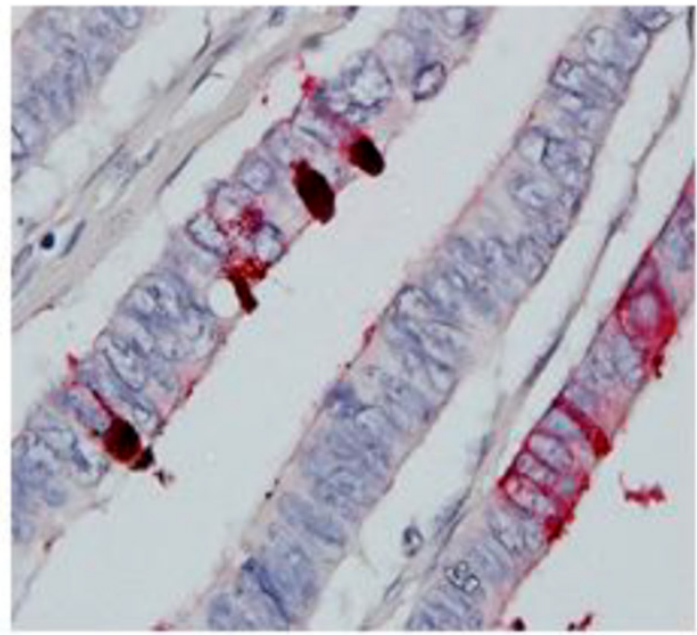

Oil 100X

Fig 3. Under high power, one sees antigen shed into the bowel lumen to eventually be incorporated in the stool.

\section{NEO-151 Antigen Detection in Stool}

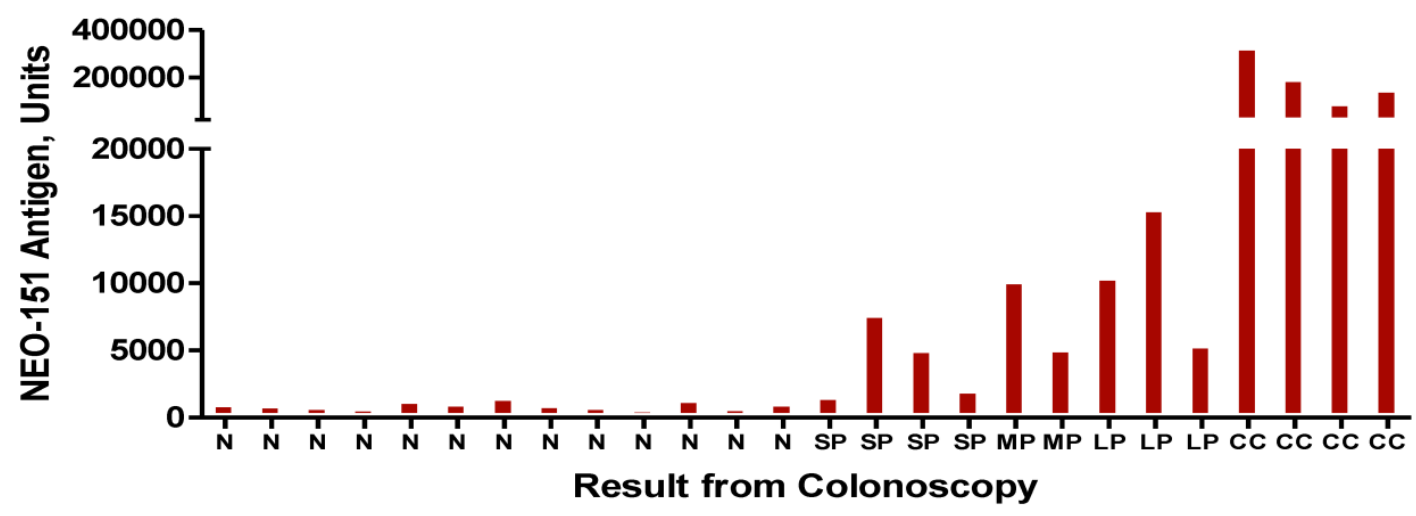

Fig 4. Results of stool test with $\mathrm{N}$ representing -normal patients, SP- small polyp, LP-large polyp and CC- colon cancer. The units are for the most part measured in nannogms. of antigen detected by the ELISA procedure.

\section{Competing Interests}

The authors have declared that no competing interest exists.

\section{References}

1. Damania D., Roy H.K., Subramanian H., Weinberg D.S., Rex D.K., Goldberg M.H., Muldoon J., Cherkezyan L., Zhu Y., Bianchi L.K., Shah D., Pradhan P., Borkar M., Lynch H., and Backman V. Nanocytology of rectal colonocytes to assess risk of colon cancer based on field cancerization. Cancer Res 2012; 72(11): 2720-7.
2. Hollinshead A., Elias G., Arlen M., Buda B., Mosley M, Scherrer J. Specific Active Immunotherapy in patients with adenocarcinoma of the colon utilizing tumor associated antigens (TAA). Cancer 1985;56 (3): 480-489.

3. Arlen M., Tsang K. The nature of the Monoclonal Antibodies derived from Immunogenic membrane antigens of human colon carcinoma origin J. Tumor Marker Oncology 1990; 5: 313-319.

4. Arlen M, Arlen P, Tsang A, Wang X, Gupta R. The Therapeutic Value of Monoclonal Antibodies Directed Against Immunogenic Tumor Glycoproteins. J Cancer 2010; 1:209-222.

5. Arlen M., Wang X., Luka J., Gupta R., Saric O. Arlen P. The Use of Specific Monoclonal Antibodies to Target Immunogenic Tissue Membrane Proteins in Patients with Recurrent Pancreatic and Colon Cancer. Cancer Drug Delivery 2012; 9:52-56. 